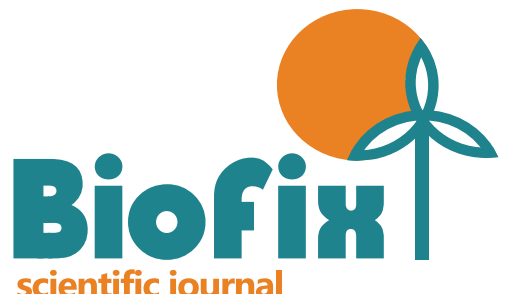

scientific journal

v. 2 n. 12017

\section{COMPARAÇÃO DE MÉTODOS DE QUANTIFICAÇÃO DE CARBONO EM BRACATINGA (Mimosa scabrella Benth.)}

\author{
COMPARISON OF CARBON QUANTIFICATION METHODS IN BRACATINGA
}

(Mimosa scabrella Benth.)
Recebido em 25/01/2017

Aceito em 21/02/2017

Publicado em 20/04/2017

DOI: dx.doi.org/10.5380/biofix.v2i1.50329

\author{
Ana Paula Marques Martins ${ }^{1}$ \\ Renata Reis de Carvalho ${ }^{2}$ \\ Clarice de Andrade ${ }^{3}$ \\ Carlos Roberto Sanquetta ${ }^{4}$
}

\begin{abstract}
Universidade Federal do Paraná, Curitiba, Paraná, Brasil anapaula_marquesm@yahoo.com.br ${ }^{1}$,claricedeandrade2@gmail.com ${ }^{3}$ \& carlos_sanquetta@hotmail.com ${ }^{4}$

Universidade Estadual do Centro-Oeste, Irati, Paraná, Brasil renatacarvalho88@gmail.com²
\end{abstract}

\section{RESUMO}

O presente trabalho teve como objetivo comparar métodos de quantificação de carbono em bracatinga. Foi determinada a biomassa fresca do fuste, galhos, folhas e raízes de 30 árvores provenientes do município de General Carneiro, estado do Paraná, e convertida em seca após análises em laboratório. Os teores de carbono (TC) foram determinados no equipamento LECO C-144, ao passo que os estoques individuais de carbono foram obtidos pela multiplicação das biomassas secas pelos respectivos TC. Foram calculados os fatores de expansão de biomassa (FEB) e a razão de raízes (R). Os métodos de estimativa de carbono analisados foram: biomassa do fuste (bf) combinada com FEB, R e teor médio de carbono (TCméd); bf combinada com FEB, R e teor ponderado de carbono (TCpond); e equações alométricas de simples, dupla e tripla entradas. Obteve-se os valores médios de $\mathrm{FEB}=1,59$ e $\mathrm{R}=0,15$, indicando que $59 \%$ da biomassa aérea correspondem à copa e $15 \%$ da biomassa total são provenientes das raízes. Não houve diferença estatística significativa entre as médias das metodologias de estimativas de carbono. A equação alométrica 3 , que utilizou as variáveis dap, altura total e densidade, foi a que apresentou o melhor desempenho, porém, a equação 1 , com apenas o dap como variável independente, propiciou resultados semelhantes. De acordo com as estatísticas adicionais, concluiu-se que as equações propiciaram os melhores indicadores; seguidas pelas metodologias que empregam os teores de carbono ponderado e carbono médio.

PALAVRAS-CHAVE: Equação alométrica, Fator de expansão de biomassa, Razão de raízes.

\section{ABSTRACT}

This study aimed to compare methods for quantifying carbon in bracatinga. Fresh biomass was determined for stem, branches, leaves, and roots of 30 trees from General Carneiro, Paraná State, and converted to dried after laboratory analysis. Carbon contents (TC) were determined in LECO C-144 equipment, while the individual carbon stocks were obtained by multiplying the dry biomass by respective TC. Biomass expansion factor (FEB) and rootto-shoot ratio $(R)$ were calculated. Carbon estimation methods were analyzed: stem biomass (bf) with FEB, $R$, and mean carbon content (TCméd); bf with FEB, R, and carbon weighted content (TCpond); and allometric equations of single, double, and triple entries. Difference between methods was analyzed by analysis of variance and additional statistics. Mean values of $F E B=1.59$ and $R$ $=0.15$ were obtained, indicating $59 \%$ of the biomass corresponding to canopy, and $15 \%$ of the total biomass are roots. There was nonsignificance difference between carbon estimates methodologies. Allometric equation 3 , which used the variables dap, total height, and density, showed the best performance, however, equation 1 , with only dap as an independent variable, showed similar results. According to the additional statistics, we conclude the equations have provided best indicators, followed by the methods weighted carbon, and mean carbon contents.

KEYWORDS: Allometric equation, Factor expansion biomass, Rootto-shoot ratio. 


\section{INTRODUÇÃO}

A espécie Mimosa scabrella Benth, conhecida popularmente como bracatinga ou amendola, é uma espécie pioneira com distribuição nas montanhas e planaltos dos estados da região Sul do Brasil. É característica e exclusiva das regiões da Floresta Ombrófila Mista, com ocorrência descontínua em vegetação primária, porém, abundante em formações secundárias, em áreas que já devastadas por ações antrópicas, que sofreram queimadas e com iluminação abundante. Com isso, a espécie forma os chamados bracatingais, consideradas associações puras e dispersas por vastas áreas do planalto sulino (CYSNEIROS et al., 2015; MACHADO et al., 2015).

O uso da madeira dessa espécie é bastante versátil, podendo ser utilizada para madeira serrada, celulose, painéis e como facilitadora para recuperação de ambientes degradados (CARVALHO 2002). Apesar dessa versatilidade, o uso da madeira é basicamente destinado para o mercado de lenha há mais de 100 anos, devido ao reconhecido potencial energético da espécie (SILVA et al., 2006; MACHADO et al., 2015). A espécie tem pouca exigência por tratos silviculturais, sendo um importante fator para o seu manejo, por estar aliada ao rápido crescimento e a rusticidade (DOSSA, 2004).

Ademais, os bracatingais são importantes fixadores de carbono, uma vez que, durante a permanência desses, o carbono é removido da atmosfera e estocado em suas estruturas (CALDEIRA et al., 2003). Desse modo, o conhecimento sobre a capacidade das florestas em armazenar carbono torna-se importante, visto que é possível implantar reflorestamentos visando à mitigação das mudanças climáticas e o desenvolver projetos de remoção de gases de efeito estufa (GORGENS et al., 2005; WEBER et al., 2006).

As estimativas de biomassa e carbono em florestas podem ser realizadas por métodos diretos e indiretos, sendo que os métodos diretos consistem em mensurar toda a biomassa, geralmente por meio de um processo destrutivo (SANQUETTA, 2002). Contudo, essa abordagem torna-se inviável para a aplicação em extensas áreas, devido ao custo e ao tempo de execução. De outro modo, os métodos indiretos visam estimativas por meio de modelagem da biomassa e do carbono, os quais utilizam fatores e/ou equações. Contudo, tais fatores e equações necessitam serem ajustados por meio de uma base de dados, o que garante a validade das estimativas (SANQUETTA et al., 2014a).

De acordo com Soares e Tomé (2004), equações de regressão com o carbono (C) como variável dependente, além de variáveis dendrométricas (diâmetro e altura, por exemplo) como independentes, são comumente denominadas de equações alométricas. Essas equações geralmente são recomendáveis por oferecerem estimativas diretas de carbono em função das variáveis dendrométricas, as quais são de fácil mensuração em campo. Contudo, quando as equações alométricas não podem ser empregadas, seja por ausência ou má qualidade dos dados, é possível o uso de fatores de expansão específicos para diferentes faixas etárias dos povoamentos florestais.

Dentre esses fatores de expansão, pode-se citar o fator de expansão de biomassa (FEB) e a razão de raízes (R) para quantificar o carbono nas florestas (CORTE et al., 2012). Segundo Sanquetta et al. (2014a), o FEB é um fator que extrapola a massa do fuste da árvore para toda a biomassa aérea, considerando os galhos e a folhagem. Esse fator geralmente calcula o estoque da biomassa aérea com base apenas no volume e na densidade da madeira. Por outro lado, $\mathrm{R}$ calcula a biomassa de toda $\mathrm{a}$ árvore por meio da razão entre a biomassa subterrânea (raízes) e a biomassa aérea (SANQUETTA et al., 2014a). Assim, o objetivo deste estudo foi comparar métodos de quantificação de carbono em bracatinga ( $M$. scabrella) provenientes do município de General Carneiro, no estado do Paraná.

\section{MATERIAL E MÉTODOS}

Para a presente pesquisa, foram coletados dados de 30 árvores de $M$. scabrella de bracatingais localizados no município de General Carneiro, estado do Paraná. O clima da região de estudo caracteriza-se como Cfb (Köppen), com verões frescos e sem estação seca definida, temperatura média do mês mais frio inferior a $17^{\circ} \mathrm{C}$, e no mês mais quente inferior a $22^{\circ} \mathrm{C}$ (IAPAR, 2017).

As árvores foram selecionadas de acordo com a distribuição diamétrica, sendo mensuradas, cortadas e separadas nos compartimentos: fuste, galhos, casca e folhas, com a quantificação das raízes, foi realizado a escavação até $1,0 \mathrm{~m}$ de profundidade. A biomassa fresca foi determinada em campo com o auxílio de uma balança de gancho. Para cada compartimento, foi retirada uma amostra de $200 \mathrm{~g}$ e encaminhada para laboratório, as quais foram pesadas, secas em estufa a $70^{\circ} \mathrm{C}$ e moídas em moinho tipo Willey. Com as amostras moídas, foi realizada a análise dos teores de carbono no equipamento LECO C-144.

A biomassa aérea seca total (ba) de cada árvore foi 
calculada a partir das relações entre as biomassas secas e frescas das amostras. O Fator de Expansão da Biomassa (FEB) e a Razão de Raízes (R) foram calculados utilizando as seguintes expressões:

$$
\begin{aligned}
& F E B=\frac{b a}{b f} \\
& R=\frac{b r}{b a}
\end{aligned}
$$

Em que: ba = biomassa seca aérea $(\mathrm{kg})=\mathrm{bf}+\mathrm{bg}+\mathrm{bfol}$ $+\mathrm{bc} ; \mathrm{bf}=$ biomassa seca do fuste sem casca $(\mathrm{kg}) ; \mathrm{bg}=$ biomassa seca dos galhos ( $\mathrm{kg})$; bfol = biomassa seca das folhas (kg); bc biomassa das cascas (kg); e br = biomassa seca das raízes $(\mathrm{kg})$.

Adicionalmente, foram ajustadas três equações alométricas usualmente utilizadas em trabalhos similares e disponíveis na literatura:

Eq. $1 \log \left(C_{\text {tot }}\right)=\beta_{0}+\beta_{1} \log ($ dap $)$

Eq. $2 \log \left(C_{\text {tot }}\right)=\beta_{0}+\beta_{1} \log ($ dap $)+\beta_{2} \log (h t)$

Eq. $3 \log \left(C_{\text {tot }}\right)=\beta_{0}+\beta_{1} \log ($ dap $)+\beta_{2} \log (h t)+\beta_{3} \log (\rho)$

Em que: Ctot $=$ carbono total $(\mathrm{Kg})$; dap $=$ diâmetro à altura do peito $(\mathrm{cm}) ; \mathrm{ht}=$ altura total $(\mathrm{m}) ; \rho=$ densidade $\left(\mathrm{g} / \mathrm{cm}^{3}\right) ; \log =$ logaritmo neperiano; e $\beta 0, \beta 1, \beta 2$ e $\beta 3=$ coeficientes de regressão.

O desempenho das equações alométricas para estimativa de carbono total foi analisado segundo critérios de ajuste e seleção de modelos de regressão, como: erro padrão da estimativa relativo (Syx\%); coeficiente de determinação ajustado ( $\left.R^{2} a j\right)$; e análise da dispersão gráfica dos resíduos.

Dois teores de carbono foram calculados: TCméd, que consiste no valor da média aritmética de todos os 150 teores de carbono (5 compartimentos x 30 árvores); e TCpond, sendo o valor da média ponderada dos valores individuais de teores de carbono com base nas biomassas de cada compartimento.

Assim, foram utilizadas cinco metodologias para estimar o carbono:

1. Carbono baseado na biomassa do fuste (bf) combinada com FEB, $\mathrm{R}$ e teor médio de carbono (TCméd);

2. Carbono baseado na bf combinada com FEB, R e teor ponderado de carbono (TCpond);

3. Equação alométrica com dap como variável independente;
4. Equação alométrica com dap e ht como variáveis independentes;

5. Equação alométrica com dap, ht e $\rho$ como variáveis independentes.

Para verificar a existência de diferenças estatisticamente significativas entre as metodologias, foi realizada a Análise de Variância (ANOVA) para um delineamento inteiramente ao acaso (DIC), sendo previamente aplicado o teste de Bartlett para verificar a homogeneidade das variâncias. Para isso, cada metodologia foi considerada como um tratamento, em que o valor real de carbono foi tratado como testemunha. Quando constatadas diferenças estatísticas significativas entre as médias, foi realizado o teste de comparação de médias de Tukey. Todos os testes estatísticos foram executados com o auxílio do suplemento Action Stat 3.0.2 no Microsoft Excel, utilizando o nível de significância de $5 \%$.

Para avaliar a acurácia de cada método de estimativa do carbono total para M. scabrella, foram calculadas as estatísticas complementares de desvio (D), desvio padrão das diferenças (SD), soma do quadrado do desvio relativo (SQRR) e porcentagem de resíduos (RP), além da análise gráfica dos resíduos. Para correção da discrepância logarítmica, foi utilizado o Fator de Meyer (FM).

$$
\begin{aligned}
& D=\left(\sum \text { Diff }_{i}\right) / N \\
& S D=\left[\sum\left(\text { Diff }_{i}-c_{i}\right)^{2} /(N-1)\right]^{0,5} \\
& S Q R R=\sum\left(\text { Diff }_{i} / c_{i}\right)^{2} \\
& R P=\sum\left(\text { Diff }_{i} / c_{i}\right) * 100 / N \\
& F M=e^{(0,5 * \text { Syx })^{2}}
\end{aligned}
$$

Em que: Diffi = desvios do carbono $(\mathrm{kg}) ; \mathrm{ci}=$ carbono total observado $(\mathrm{kg}) ; \mathrm{N}=$ número de árvores; $\mathrm{e}=$ exponencial; e Syx = erro padrão da estimativa.

Para determinar qual método apresentou maior acuracidade nas estimativas, foi aplicado uma ordenação do desempenho de cada metodologia em relação a cada estatística complementar na Tabela 1 , atribuindo nota 1 para o de melhor desempenho e nota 5 para o pior, ao passo que valores iguais receberam notas idênticas. Posteriormente, foi realizado o somatório dos valores, cujo método com menor pontuação foi considerado o de melhor desempenho na estimativa de carbono total. 


\section{RESULTADOS E DISCUSSÃO}

Na Tabela 1 é possível observar as estatísticas descritivas para o fator de expansão de biomassa (FEB), razão de raízes $(\mathrm{R})$, teor de carbono médio (TC médio) e teor de carbono ponderado (TC ponderado), além dos parâmetros e das estatísticas de ajuste e seleção para as três equações alométricas.

Tabela 1. Grandezas calculadas e estimadas para quantificação do carbono de Mimosa scabrella

\begin{tabular}{ccccccc}
\hline Grandeza & Média & DP & CV\% & Eq 1 & Eq 2 & Eq 3 \\
\hline FEB & 1,592 & 0,284 & 17,82 & - & - & - \\
$R$ & 0,153 & 0,040 & 26,35 & - & - & - \\
TC médio & 0,447 & 0,012 & 2,69 & - & - & - \\
$T C$ pond. & 0,440 & 0,013 & 2,95 & - & - & - \\
$\beta_{0}$ & - & - & - & $-1,3271$ & $-1,6918$ & $-2,7130$ \\
$\beta_{1}$ & - & - & - & 2,5859 & 2,2339 & 2,4989 \\
$\beta_{2}$ & - & - & - & - & 0,6596 & 0,0765 \\
$\beta_{3}$ & - & - & - & - & - & 0,5301 \\
$R_{a j}^{2}$ & - & - & - & 0,9684 & 0,9839 & 0,9935 \\
$S y x \%$ & - & - & - & 5,53 & 4,64 & 2,85 \\
\hline
\end{tabular}

Para o presente estudo, nota-se que o valor médio de FEB foi 1,592 e $R$ foi 0,153 , ou seja, $59 \%$ da biomassa total correspondem à parte aérea e $15 \%$ às raízes. Observa-se que houve variação de $17,82 \%$ e $26,35 \%$ em torno da média para esses fatores, em decorrência da variabilidade em dap dos indivíduos mensurados, com amplitude de 5 a $20 \mathrm{~cm}$. Silveira (2010) afirma que existe grande variação dos valores de FEB entre classes de dap, em que ainda não há um padrão para o FEB, uma vez que é influenciada por outros fatores além da biomassa.

Mognon et al. (2013), ao realizarem a modelagem do estoque de carbono por meio de modelos alométricos para a $M$. scabrella, encontraram valores médios iguais a 1,41 para FEB e 0,18 para $R$, sendo semelhantes aos encontrados na presente pesquisa. Corte et al. (2012) obtiveram, para Pinus sp. no Sul do Brasil, valores de FEB igual 1,47 e de $R$ igual a 0,17 , o que indica que $47 \%$ da biomassa aérea correspondem à copa e $17 \%$ da biomassa total são raízes.

Levy et al. (2010), em estudo para coníferas, obtiveram valor de FEB entre 1,04 e 2,32, e para $R$ igual a 0,36 . Sanquetta et al. (2014a) observaram FEB variando de 1,05 a 2,06 para Araucaria angustifolia, o que indicou que há árvores com pouca biomassa de copa. Enquanto $\mathrm{R}$ variou de 0,03 a 0,12, mostrando pouca biomassa subterrânea. Para os valores de FEB encontrados no presente estudo, é possível afirmar que são semelhantes aos encontrados na literatura.

O plantio da espécie $M$. scrabella apresentou teores de carbono médio e carbono ponderado iguais a 0,447 e 0,440 , respectivamente. Rochadelli (2001), após estudar a estrutura de fixação de carbono em bracatingais, obteve os teores relativos de carbono orgânico fixado na biomassa total entre 40 e 45\%. Silva et al. (2015), trabalhando com o estoque de carbono na biomassa aérea em Eucalyptus spp., obtiveram o teor de carbono em termos médios presente na biomassa total da árvore de $46,3 \%$. Sanquetta et al. (2014b) avaliaram os teores e os estoques de carbono da espécie Acacia mearnsii no estado do Rio Grande do Sul e relataram que o teor médio de carbono variou de $45,01 \%$ a $46,50 \%$. Portanto, os valores encontrados no presente estudo estão condizentes com os de outros pesquisadores.

Quanto às equações alométricas, observa-se que as estatísticas de ajuste e seleção de modelos melhoram com a inclusão de variáveis independentes, sendo que os melhores resultados foram obtidos para a equação 3 (Figura 1), a qual usa dap, ht e $\rho$ para estimar o carbono total. Porém, nota-se que a melhoria na acuracidade da estimativa de carbono não é tão expressiva quando se considera as demais equações.

Dessa forma, é possível afirmar que, considerando a facilidade de coleta das variáveis independentes, a equação 3 propiciou o melhor desempenho estatístico. Porém, a equação 1 tem aplicação mais prática, por necessitar apenas no conhecimento do dap da árvore para estimar o carbono total, uma vez que seu desempenho estatístico apresenta resultados satisfatórios quando comparada às demais equações ajustadas.

Para verificar a homogeneidade de variâncias, foi realizado o teste de Bartlett (Tabela 2), em que é possível observar que as variâncias dos tratamentos não apresentam diferenças estatisticamente significativas ( $p$ valor 0,999).

Tabela 2. Teste de homogeneidade de variâncias de Bartlett para metodologias de estimativa de carbono para M. scabrella

\begin{tabular}{cc}
\hline Informação & Valor \\
\hline Estatística do teste & 0,1508 \\
Graus de liberdade & 5 \\
p-valor & 0,9996 \\
\hline
\end{tabular}




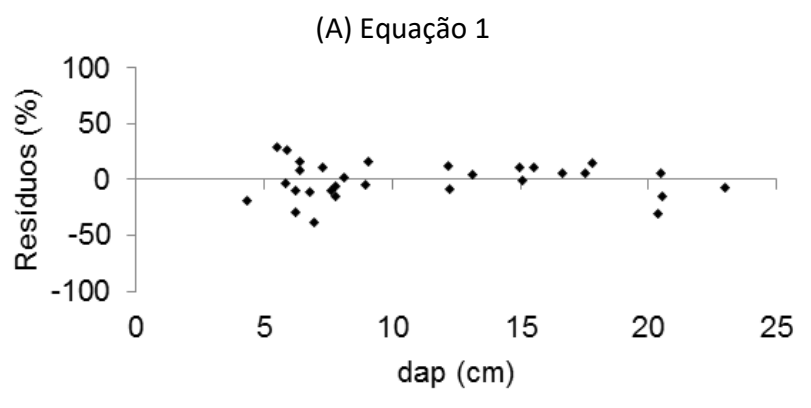

(B) Equação 2
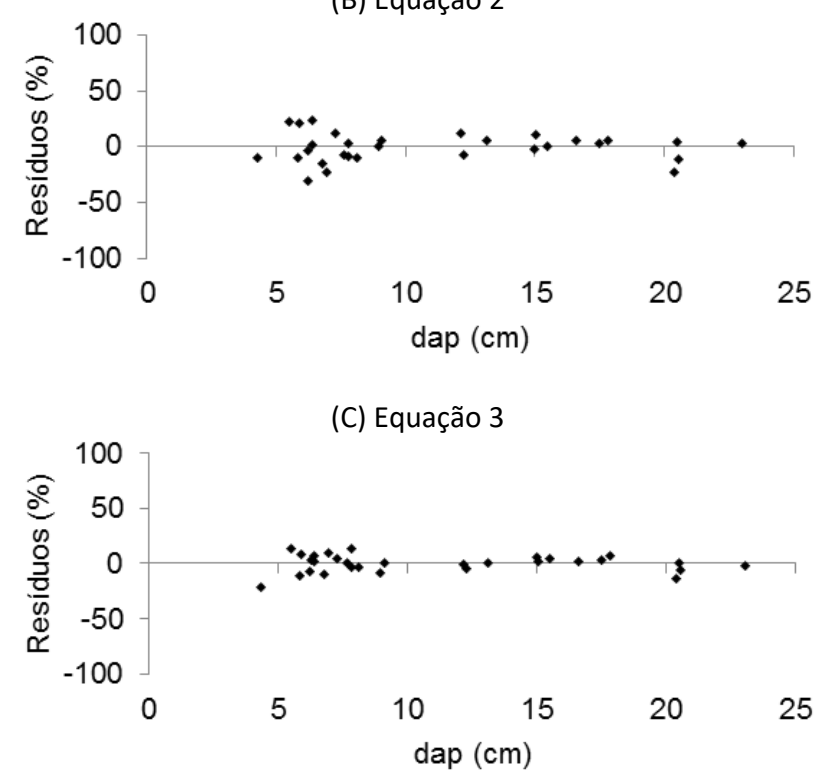

Figura 1. Dispersão gráfica dos resíduos das equações alométricas para estimativa do carbono total para M. scrabella.

Assim sendo, foi possível a aplicação da ANOVA (Tabela 3), na qual não foi verificada a existência de diferenças estatisticamente significativas entre as metodologias para a estimativa do carbono total de $M$. scabrella (p-valor 0,999), não sendo necessária a aplicação do teste de comparação de médias de Tukey.

Tabela 3. Análise de variância para metodologias de estimativa de carbono para $M$. scabrella

\begin{tabular}{cccccc}
\hline F.V. & G.L. & S.Q. & Q.M. & F & p-valor \\
\hline Fator & 5 & 53,67 & 10,737 & 0,0063 & 0,999 \\
Resíduos & 174 & 298975,99 & 1718,253 & & \\
\hline
\end{tabular}

F.V. = Fonte de Variação; G.L. = graus de liberdade; S.Q. = Soma de Quadrados; e Q.M. = Quadrado Médio.

Comparando a acuracidade dos cinco métodos de estimativa de carbono testados no presente estudo (Tabela 4) por meio das estatísticas complementares, foi possível constatar a menor eficiência do método baseado na biomassa do fuste (bf) combinada com FEB, R e teor médio de carbono (TCméd), devido aos maiores valores modulares de desvio padrão das diferenças, soma do quadrado do resíduo relativo e porcentagem dos resíduos.

Tabela 4. Desvio (D), desvio padrão das diferenças (SD), soma do quadrado do resíduo relativo (SQRR) e porcentagem dos resíduos (RP) para a estimativa do carbono total para Mimosa scabrella

\begin{tabular}{cccccc}
\hline Modelo & $\boldsymbol{D}$ & $\boldsymbol{S D}$ & SQRR & $\boldsymbol{R P}$ & Ranking \\
\hline TCpond & $-5,731$ & 62,637 & 3,709 & $-18,638$ & 4 \\
TCméd & $-6,575$ & 63,973 & 4,232 & $-20,626$ & 5 \\
Equação 1 & $-1,446$ & 57,212 & 2,303 & $-5,832$ & 3 \\
Equação 2 & $-1,216$ & 56,010 & 2,890 & $-5,996$ & 2 \\
Equação 3 & $-1,339$ & 56,288 & 2,035 & $-5,058$ & 1 \\
\hline
\end{tabular}

As equações alométricas ajustadas apresentaram boa precisão nas estimativas, gerando estatísticas de ajuste superiores e reduzidos valores de desvio padrão das médias, soma dos quadrados dos resíduos relativos e porcentagem dos resíduos. As mesmas estatísticas foram utilizadas por Martins et al. (2015) de forma complementar para comparação e seleção de metodologias de estimativa de volume total para Araucaria angustifolia, sendo utilizadas como critério definidor do método mais adequado.

\section{CONCLUSÕES}

Não há diferença estatística entre as médias das metodologias de estimativas de carbono para $M$. scabrella. Dentre as equações alométricas ajustadas, a equação 3 , a qual utiliza o maior número de variáveis independentes, propicia melhores resultados, porém a equação 1 , que utiliza dap como variável independente, acarreta em resultados semelhantes. Desse modo, a equação 1 é a mais indicada, por proporcionar maior facilidade na coleta de dados.

Considerando as estatísticas complementares de avaliação da acuracidade das estimativas, as equações alométricas propiciam os melhores indicadores, seguidas da metodologia que emprega o teor de carbono ponderado, e, por último, a do teor de carbono médio.

\section{REFERÊNCIAS}

CALDEIRA, M. V. W.; SCHUMACHER, M. V.; BARICHELLO, L. R.; VOGEL, H. L. M. Determinação de carbono orgânico em 
povoamentos de Acacia mearnsii de Wild. plantados no Rio Grande do Sul. Revista Acadêmica: Ciências Agrárias e Ambientais, v. 1, n. 2, p. 47-54, 2003.

CARVALHO, P. E. R. Bracatinga. Colombo: Embrapa Florestas, 2002. 12 p.

CORTE, A. P. D.; SILVA, F.; SANQUETTA, C. R. Fator de expansão de biomassa e razão de raízes - parte aérea para Pinus spp. plantadas no Sul do Brasil. Floresta, v. 42, n. 4, p. 755-768, 2012.

CYSNEIROS, V. C.; MARTINS, A. P. M.; SCHMIDT, L. N.; ACCIOLY, Y.; MACHADO, S. A. Eficiência do método dos dois diâmetros para a estimativa volumétrica de Mimosa scabrella Benth. In.: SIMPÓSIO LATINO-AMERICANO SOBRE MANEJO FLORESTAL, 6. 2015, Santa Maria, RS. Anais... Santa Maria, RS, 2015. p. 301306.

DOSSA, D. Cenário sócio-econômico da produção de bracatinga. In: OFICINA SOBRE BRACATINGA NO VALE DA RIBEIRA, 1, 2004, Curitiba. Anais... Curitiba, 2004. p. 5-12.

GORGENS, E. B.; OLIVEIRA, M. L. R.; LEITE, H. G.; BRUM NETO, M. S. Metodologia para Monitoramento do Estoque de Carbono: Revisão e Estudo de Caso. Biomassa \& Energia, v. 2, n. 2, p. $169-$ 175, 2005.

IAPAR - INSTITUTO AGRONÔMICO DO PARANÁ. Cartas climáticas do Paraná: classificação climática. 2017. Disponível em <http://www.iapar.br/modules/conteudo/conteudo.php?co nteudo $=863>$. Acesso em 20/02/ 2017.

LEVY, P. E.; HALE, S. E.; NICOLI, B. C. Biomass expansion factors and root: shoot ratios for coniferous tree species in Great Britain. Forestry, v. 77, n. 5, p. 421-430. 2010.

MACHADO, S. A.; SOUZA, R. F.; APARECIDO, L. M. T.; RIBEIRO, A.; CZELUSNIAK, B. H. Evolução das variáveis dendrométricas da bracatinga por classe de sítio. Cerne, v. 21, n. 2, p. 199-207, 2015.

MARTINS, A. P. M.; SCHMIDT, L. N.; CYSNEIROS, V. C.; MACHADO, S. A.; FIGUEIREDO FILHO, A. Comparison of methods for estimating total volume of Araucaria angustifolia (Bertol.) Kuntze. Australian Journal of Basic and Applied Sciences, v. 9, n. 35, p. 239-244, 2015.

MOGNON, F.; DALLAGNOL, F. S.; DOUBRAWA, B.; FLIZIKOWSKI, L. C.; SANQUETTA, C. R. Modelagem do estoque de carbono para Mimosa scabrella Benth. localizadas no Paraná. Revista Acadêmica: Ciências Agrárias e Ambientais, v. 11, n. 1, p. 121129, 2013.

ROCHADELLI, R. A estrutura de fixação dos átomos de carbono em reflorestamento: estudo de caso: Mimosa scabrella Bentham, Bracatinga. 2001. 86 f. Tese (Doutorado em Engenharia Florestal), Universidade Federal do Paraná, Curitiba, 2001.

SANQUETTA, C. R. Metodologias para determinação de biomassa florestal. In: SANQUETTA, C. R.; WATZLAWICK, L. F.; BALBINOT, R.; ZILIOTTO, M. A. B.; GOMES, F. S. As florestas e o carbono. Curitiba: FUPEF/ Imprensa da UFPR, 2002. 264 p.
SANQUETTA, C. R.; CORTE, A. P. D.; MOGNON, F.; MAAS, G. C. B.; RODRIGUES, A. L. Estimativa de carbono individual para Araucaria angustifolia. Pesquisa Agropecuária Tropical, v. 44, n. 1, p. 1-8, 2014a.

SANQUETTA, C. R.; BEHLING, A.; CORTE, A. P. D.; SIMON, A.; PSCHEIDT, H.; RUZA, M. S.; MOCHIUTTI, S. Estoques de biomassa e carbono em povoamentos de acácia negra em diferentes idades no Rio Grande do Sul. Scientia Forestalis, v. 42, n. 103, p. 361-370, 2014b.

SILVA, V. P. da; MAZUCHOWSKI, J. Z.; PINTO, A. F.; MINIOLLI NETTO, J.; BECKER, J. C. Memórias da oficina sobre bracatinga no Vale do Ribeira: 2004. Curitiba, PR. Colombo: Embrapa Florestas, 2006. 107 p.

SILVA, C. A.; KLAUBERG, C.; CARVALHO, S. P. C.; M. C. PICCOLO, M. C.; RODRIGUEZ, L. C. E. Estoque de carbono na biomassa aérea florestal em plantações comerciais de Eucalyptus spp. Scientia Forestalis, v. 43, n. 105, p. 135-146, 2015.

SOARES, P.; TOMÉ, M. Analysis of the effectiveness of biomass expansion factors to estimate stand biomass. In: HASENAUER, H.; MAKELA, A. Modeling forest production. Viena: BOKU University of Natural Resources and Applied Life Sciences, 2004. p. 368-374.

WEBER, K. S.; BALBINOT, R.; WATZLAWICK, L. F.; SANQUETTA, C. $R$. Teores de carbono orgânico de seis espécies naturais do ecossistema da Floresta Ombrófila Mista. Ambiência, v. 2, n. 2, p. 167-177, 2006. 